\title{
Epidemiological approach to nosocomial infection surveillance data: the Japanese Nosocomial Infection Surveillance System
}

\author{
Machi Suka $\cdot$ Katsumi Yoshida $\cdot$ Jun Takezawa
}

Received: 11 May 2007/Accepted: 9 August 2007/Published online: 11 December 2007

(C) The Japanese Society for Hygiene 2008

\begin{abstract}
Surveillance of nosocomial infection is the foundation of infection control. Nosocomial infection surveillance data ought to be summarized, reported, and fed back to health care personnel for corrective action. Using the Japanese Nosocomial Infection Surveillance (JANIS) data, we determined the incidence of nosocomial infections in intensive care units (ICUs) of Japanese hospitals and assessed the impact of nosocomial infections on mortality and length of stay. We also elucidated individual and environmental factors associated with nosocomial infections, examined the benchmarking of infection rates and developed a practical tool for comparing infection rates with case-mix adjustment. The studies carried out to date using the JANIS data have provided valuable information on the epidemiology of nosocomial infections in Japanese ICUs, and this information will contribute to the development of evidence-based infection control programs for Japanese ICUs. We conclude that current surveillance systems provide an inadequate feedback of nosocomial infection surveillance data and, based on our results, suggest a methodology for assessing nosocomial infection surveillance data that will allow infection control
\end{abstract}

This article is based upon the research that was given encouragement award at the 77th annual meeting of the Japanese Society for Hygiene held in Osaka, Japan on 25-28 March 2007.

M. Suka $(\bowtie) \cdot$ K. Yoshida

Department of Preventive Medicine, St Marianna University

School of Medicine, 2-16-1 Sugao, Miyamae-ku Kawasaki,

Kanagawa 216-8511, Japan

e-mail: suka@marianna-u.ac.jp

J. Takezawa

Department of Emergency and Intensive Care Medicine,

Nagoya University Graduate School of Medicine, Nagoya, Japan professionals to maintain their surveillance systems in good working order.

Keywords Epidemiology - Intensive care units · Japan · Nosocomial infections · Surveillance

\section{Introduction}

Infection control in the hospital setting is performed with the aim of improving the effectiveness of patient care and promoting patient safety. Infection control professionals need to recognize and explain nosocomial infections and design and implement interventions to reduce their incidence. These infection control activities should have their bases in a well-designed surveillance system of nosocomial infections [1].

Compared with the USA and other developed countries, Japan traditionally had limited sources of information on the epidemiology of nosocomial infections and, until recently, little was known about the incidence and outcome of nosocomial infections in Japanese hospitals. The Japanese Ministry of Health, Labour, and Welfare established the Japanese Nosocomial Infection Surveillance (JANIS) system in July 2000, when participating hospitals routinely started to collect and subsequently make their nosocomial infection surveillance data available for entry into a national database. The JANIS database has now become the most important source of information on the epidemiology of nosocomial infections in Japanese hospitals.

In the study reported here, we used the JANIS data to determine the incidence of nosocomial infections in intensive care units (ICUs) of Japanese hospitals and assess the impact of nosocomial infections on mortality and length of stay. We elucidated individual and environmental 
factors associated with nosocomial infections, which allowed us to identify high-risk patients and take appropriate measures to prevent infection. Infection rates are calculated as a fundamental measure for surveillance of nosocomial infections. The comparison of infection rates between hospitals and within a hospital over time contributes to the improved design, implementation and evaluation of infection control programs [2, 3]. We examined the benchmarking of infection rates and developed a practical tool for comparing infection rates with case-mix adjustment.

\section{The JANIS system}

The ICU component of the JANIS system consists of more than 30 ICUs of multidisciplinary hospitals throughout Japan that have more than 200 beds. All of the patients admitted to the participating ICUs are automatically enrolled in the survey. The following data are collected by trained physicians and nurses in each ICU and sent to the data management office by the Internet on a monthly basis: sex, age, underlying disease, severity-of-illness [4], ICU admission and discharge (date, time and route), operation (elective and urgent), device use (ventilator, urinary catheter and central venous catheter), infection (pneumonia, urinary tract infection, catheter-related bloodstream infection, sepsis, wound infection and others) and hospital discharge (date and outcome). APACHE II uses a point score based on the initial values of 12 routine physiological measurements, age and previous health status to provide a general measure of severity of illness [4]. A higher point score indicates a greater mortality risk. Infections are diagnosed according to the JANIS criteria [5], which are based on and modified from those of the National Nosocomial Infections Surveillance (NNIS) system in the USA [6]. Nosocomial (ICU-acquired) infection is defined as a newly developed infection at least 2 days after ICU admission [6]. Pathogens are classified as drug resistant or drug susceptible according to the JANIS definitions that specify a drug-resistance pattern for each pathogen [5].

The JANIS system takes various measures to establish a standardized and formatted database: a specific databaseoriented software and a written operating manual with uniform definitions and surveillance protocols are prepared in advance; workshops for data collectors are conducted on demand; reliability checks are routinely performed at the data management office. Although the participating ICUs may not represent all Japanese ICUs, the JANIS data is the only reliable source of information on the epidemiology of nosocomial infections in Japanese ICUs. Compared with the NNIS system, the JANIS system has the advantage of collecting data from each patient. The availability of the JANIS data enabled us to assess the incidence and outcome of nosocomial infections in the context of individual factors.

\section{Epidemiology of nosocomial infections in Japanese ICUs}

Incidence of nosocomial infections

Figure 1 shows the incidence of nosocomial infections in Japanese ICUs [7]. The study cohort consisted of 7374
Fig. 1 Incidence of nosocomial infections (\%) in Japanese intensive care units (ICUs) from July 2000 to May 2002. Pathogens were classified as drug resistant (filled bar) or drug susceptible (shaded bar) according to the JANIS definitions

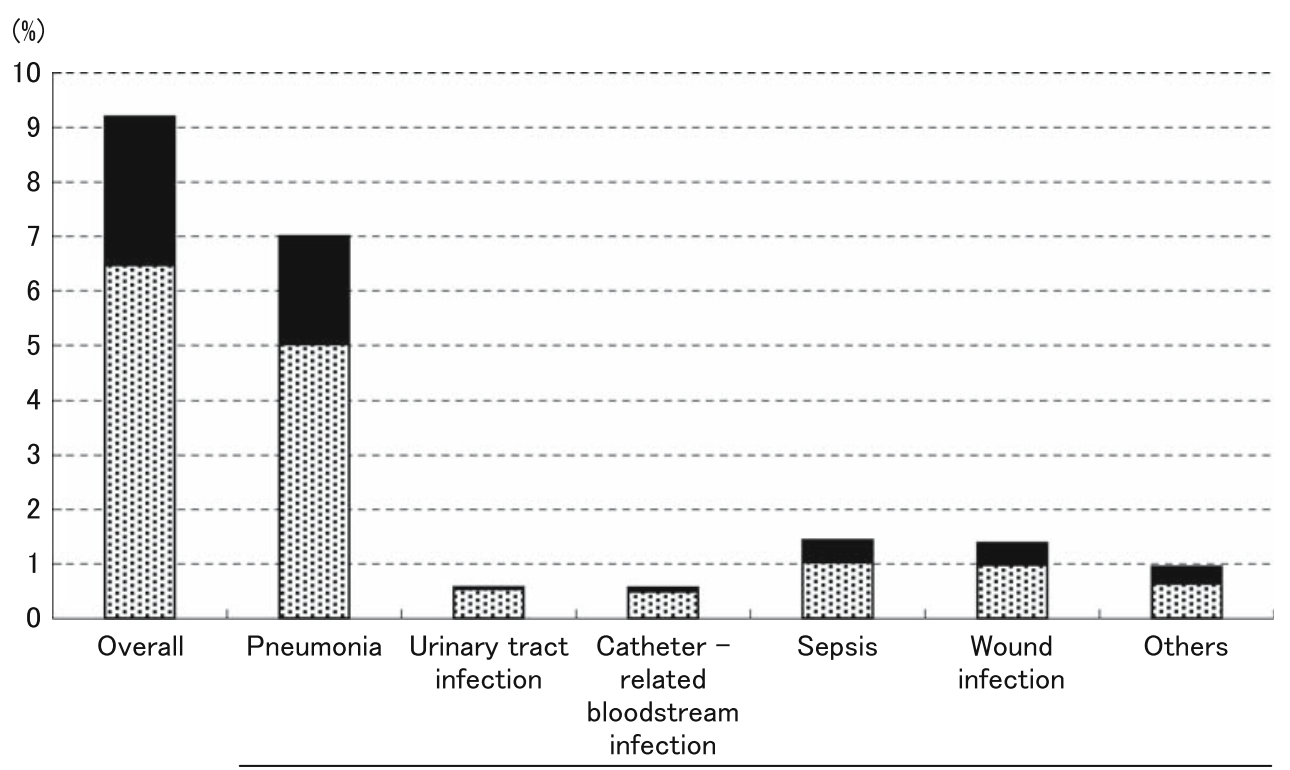

Infection type 
eligible patients, aged $\geq 16$ years, who had been hospitalized in 34 ICUs for $\geq 48 \mathrm{~h}$. Of these 7374 patients, 678 patients $(9.2 \%)$ had had at least one episode of nosocomial infection. The most common nosocomial infection was pneumonia (517 cases, 64\%), followed by sepsis (106 cases, 13\%), wound infection (102 cases, 13\%), urinary tract infection (43 cases, 5\%) and catheter-related bloodstream infection (42 cases, 5\%). Drug-resistant pathogens were detected in 201 patients with nosocomial infection (29.6\%). The majority of drug-resistant pathogens were methicillin-resistant staphylococcus aureus (MRSA).

A number of studies have been conducted to determine the incidence of nosocomial infections in ICUs. However, these studies differ considerably in terms of settings and protocols and, therefore, it is difficult to compare the reported rates accurately, although the incidence of urinary tract infection based on the NNIS data seems to be higher than that based on the JANIS data (annual reports of the JANIS system are available at: http://www.nih-janis.jp/ report/list_index.html).

\section{Outcome of nosocomial infections}

The same cohort data were analyzed to assess the impact of nosocomial infections on hospital mortality [7, 8] and length of hospital stay for survivors [8]. The crude hospital mortality in the patients with nosocomial infection $(45.8 \%$ for the cases of drug-resistant pathogens, $36.1 \%$ for the cases of drug-susceptible pathogens) was significantly higher than that in the patients without nosocomial infection (15.5\%). Multivariate analysis adjusting for sex, age, APACHE II score, operation and device use showed that the patients with nosocomial infection caused by drugresistant pathogens had a 1.4-fold higher risk of hospital mortality than those without nosocomial infection: the adjusted hazard ratio [95\% confidence interval (CI)] of nosocomial infections for hospital mortality was 1.42 (1.15-1.77) for the cases of drug-resistant pathogens and 1.11 (0.94-1.31) for the cases of drug-susceptible pathogens. The patients with nosocomial infection had a significantly longer hospital stay than the patients without nosocomial infection. After adjusting for APACHE II score, we estimated the excess length of the hospital stay $(95 \% \mathrm{CI})$ attributable to nosocomial infections to be 27.6 (18.6-36.5) days for the cases of drug-resistant pathogens and 12.8 (8.2-17.4) days for the cases of drug-susceptible pathogens.

Sepsis is a clinical syndrome describing infection and a subsequent systemic inflammatory response [9, 10]. A recent study of the JANIS data suggested that the development of sepsis leads to additional increases in mortality and length of stay among patients with nosocomial infection in Japanese ICUs [11]. In a study cohort of 20,909 eligible patients aged $\geq 16$ years, hospitalized in 28 ICUs for $\geq 24 \mathrm{~h}$, there were 928 episodes of nosocomial infection, including 168 episodes of sepsis (18.1\%). The standardized mortality ratio $(95 \% \mathrm{CI})$ was estimated at 2.43 (1.88-3.09) in those patients with nosocomial infection and subsequent sepsis and $1.18(0.82-1.21)$ in those patients with nosocomial infection only. The mean length of stay (95\% CI) following adjustment for the APACHE II score was estimated to be 15.0 (13.3-17.0) days in patients with nosocomial infection and subsequent sepsis and 11.8 (11.3-12.4) days in patients with nosocomial infection only compared with 3.8 (3.8-3.9) days in patients without nosocomial infection.

\section{Factors associated with nosocomial infections}

In order to implement interventions to reduce the incidence of nosocomial infections, infection control professionals need to understand factors associated with nosocomial infections. The identification of individual factors will allow them to identify high-risk patients, and the identification of environmental factors will allow them to take appropriate measures to prevent infection.

\section{Individual factors}

The study cohort data, consisting of 8587 eligible patients aged $\geq 16$ years who had been hospitalized in 34 ICUs for $\geq 48 \mathrm{~h}$, were analyzed to elucidate individual factors associated with nosocomial infections [12]. Table 1 shows the adjusted odds ratios for nosocomial infections. A significantly high odds ratio was found for APACHE II, urgent operation, ventilator and central venous catheter, while a significantly low odds ratio was found for women and elective operation. Although the impact of APACHE II on the incidence of nosocomial infections became weaker with longer ICU stay [13], APACHE II was recognized to be a good predictor of nosocomial infections. Patients requiring particular attention from health care workers to guard against infection are male, have a high APACHE II score, have an urgent operation and use ventilator and central venous catheter. Device use is the major changeable risk factor for nosocomial infections [14]. As mentioned above, pneumonia is the most common nosocomial infection in Japanese ICUs, and about $90 \%$ of episodes of pneumonia are associated with mechanical ventilation [15]. Improvements in the management of ventilators should be given the highest priority as a preventive measures against nosocomial infections in Japanese ICUs. 
Table 1 Adjusted odds ratios (ORs) with 95\% confidence intervals (CIs) for nosocomial infection

\begin{tabular}{lll}
\hline Individual factors & & OR $(95 \% \mathrm{CI})$ \\
\hline Sex & Men & 1.00 (Reference) \\
Age (years) & Women & $0.74(0.62-0.88)$ \\
& $16-44$ & 1.00 (Reference) \\
& $45-54$ & $0.83(0.60-1.15)$ \\
& $55-64$ & $0.83(0.62-1.12)$ \\
APACHE II & $65-74$ & $0.89(0.68-1.18)$ \\
& $75+$ & $0.75(0.56-1.00)$ \\
& $0-5$ & $1.00($ Reference) \\
& $6-10$ & $1.57(1.03-2.40)$ \\
& $11-15$ & $2.55(1.70-3.85)$ \\
Operation & $16-20$ & $3.62(2.39-5.49)$ \\
& $21-25$ & $5.38(3.50-8.27)$ \\
& $26-30$ & $5.14(3.23-8.16)$ \\
Ventilator & $31+$ & $7.09(4.34-11.59)$ \\
& None & $1.00($ Reference) \\
& Elective & $0.78(0.63-0.98)$ \\
& Urgent & $1.22(1.00-1.49)$ \\
& Nonuser & $1.00($ Reference) \\
& User & $2.11(1.62-2.76)$ \\
& Nonuser & $1.00($ Reference) \\
& User & $1.48(1.14-1.93)$ \\
\hline
\end{tabular}

Environmental factors

In 2001, 25 ICUs participating in the JANIS system took part in a questionnaire survey on ICU characteristics. The relationship between ICU characteristics and the incidence of nosocomial infections was assessed to elucidate environmental factors associated with nosocomial infections [16]. The survey identified a number of ICU characteristics associated with an increased incidence of nosocomial infections, namely, the location of the ICU close by a critical care center, resident physicians present for night duty, ICU rounds conducted by attending physicians less than once a day, case conferences held fewer than four times a month, no local guidelines for antibiotic use, common use of instruments and personnel not always using gloves for patient care. Infection control professionals should recognize the potential impacts of these factors on the incidence of nosocomial infections and consider implementing interventions to reduce the risk involved.

\section{Assessment of nosocomial infection surveillance data}

Infection rates are calculated as a fundamental measure for surveillance of nosocomial infections. They must be meaningful for comparison, either from one hospital to another or within a hospital over time [2,3]. A relatively high or increased infection rate may suggest a potential problem in the infection control program of the hospital, while a relatively low or decreased infection rate may suggest that the infection control program of the hospital is successful in preventing infection. However, the distribution of risk factors for nosocomial infections varies widely according to hospital and time. Failure to adjust adequately for case-mix will lead to erroneous conclusions [2]. Benchmarking of infection rates is important when assessing nosocomial infection surveillance data. Infection control professionals fervently hope for a practical tool that allows them to compare observed infection rates with external standards (benchmark infection rates) in the proper way.

\section{Benchmarking of infection rates}

Infection rates are calculated as the total number of episodes of nosocomial infection divided by a measure of the population at risk. The choice of the denominator is crucial for interhospital comparison [2, 3]. The JANIS data has been used to examine the distributions of several infection rates with different denominators with the aim of identifying the optimum denominator for comparing infection rates between different Japanese ICUs [17]. Figure 2 shows the distributions of infection rates (per admissions vs. per patient-days) for pneumonia of 18 Japanese ICUs. These varied widely according to the ICU, but all 18 ICUs were assessed at the same order using either infection rate. The infection rates per admissions and per patient-days were significantly correlated to each other $(r=0.99)$. Generally speaking, infection rates per patient-days are a better tool for carrying out interhospital comparison than infection rates per admissions because the former can be adjusted for length of stay [2]. It would be advisable to use infection rates per patient-days for benchmarking in order to reduce the risk of misleading.

A practical tool for comparing infection rates with case-mix adjustment

A spreadsheet was developed to calculate the standardized infection ratio (SIR) on the basis of the Japanese benchmark infection rates that were derived from the JANIS data [18]. The SIR is a well-known risk-adjusted indicator that is calculated by the indirect standardization method by dividing the total number of observed nosocomial infections by the total number of expected nosocomial infections [19]. The user of the spreadsheet inputs the number of observed nosocomial infections and patient-days 


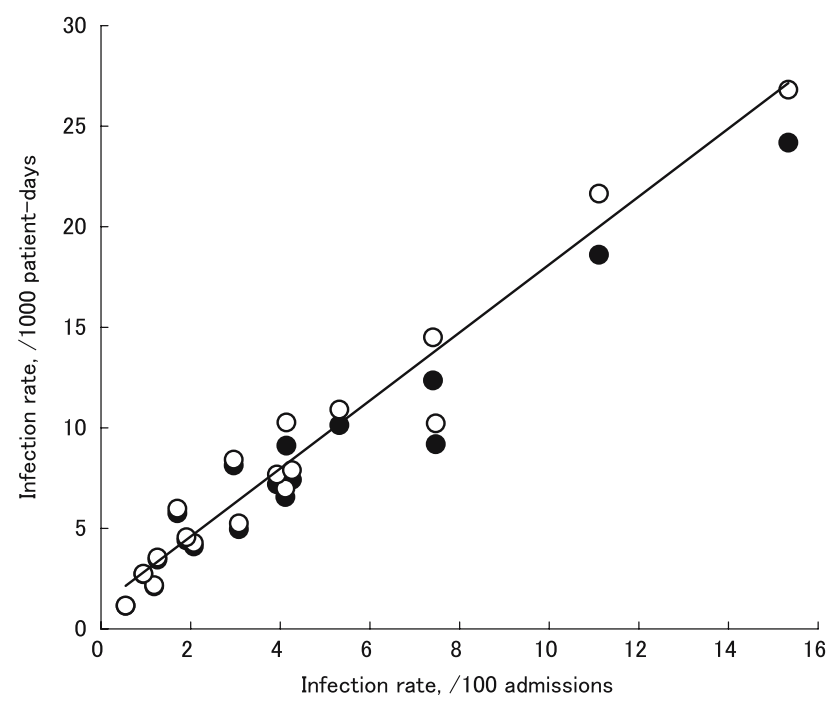

Fig. 2 Distributions of infection rates (per admissions vs. per patientdays) for pneumonia of 18 Japanese ICUs (June 2002-December 2003). Patient-days were counted as either total ICU stay (filled circle) or infection-free ICU stay (open circle)

by APACHE II, operation and ventilator, and then obtains a SIR adjusted for these three factors. A SIR value of more than one indicates that the incidence of nosocomial infections in the ICU is higher than the benchmark. Figure 3 shows SIRs of eight Japanese ICUs. For example, ICU5, ICU6 and ICU7 had a SIR of more than one in 2001. The SIR of ICU5 was gradually decreasing in 2002 and 2003, while, in contrast, those of ICU6 and ICU7 were increasing. One interpretation of these results is that ICU5 was successful in preventing infection while ICU6 and ICU7 had a potential problem in the infection control program and, therefore, should at the very least investigate the cause of the increasing incidence of nosocomial infection. The spreadsheet is simple and easy enough to be used by all infection control professionals, and it can reveal relative merits and secular changes in the incidence of nosocomial infections in the ICU. The use of the spreadsheet is expected to promote timely feedback of nosocomial infection surveillance data, which would allow infection control professionals to take prompt and efficient measures to prevent infection.

\section{Conclusion}

The studies of the JANIS data provide valuable information on the epidemiology of nosocomial infections in Japanese ICUs, and this information will contribute to the development of evidence-based infection control programs for Japanese ICUs. The primary aim of the surveillance of nosocomial infections is to introduce interventions aimed at reducing the incidence of nosocomial infections.

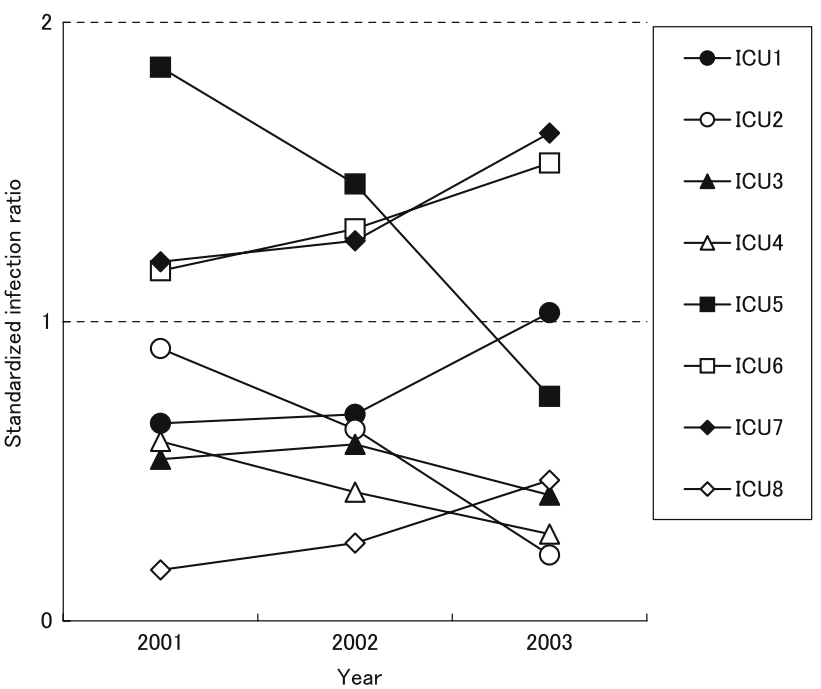

Fig. 3 Standardized infection ratios of eight Japanese ICUs. Standardized infection ratios were calculated by the indirect standardization method, which consists of dividing the total number of observed nosocomial infections by the total number of expected nosocomial infections based on the Japanese benchmark infection rates that were derived from the JANIS data

Nosocomial infection surveillance data ought to be summarized, reported and fed back to health care personnel for corrective action [1]. However, the current surveillance systems provide an inadequate feedback of nosocomial infection surveillance data. The studies of the JANIS data suggest a methodology of assessment of nosocomial infection surveillance data that will allow infection control professionals to keep the surveillance systems in good working order. For further developments of surveillance of nosocomial infections in Japanese hospitals, we may need to assess the effect of current surveillance systems on the incidence and outcome of nosocomial infections in future studies.

Acknowledgments This study was supported by the Health and Labour Sciences Research Grant (Research on Emergent and Reemerging Infectious Diseases) from the Japanese Ministry of Health Labour, and Welfare and the Grant-in-Aid for Scientific Research (Grant-in-Aid for Young Scientists 15790306 and 18790406) from the Japanese Ministry of Education, Culture, Sports, Science, and Technology.

\section{References}

1. Pittet D. Infection control and quality health care in the new millennium. Am J Infect Control. 2005;33:258-67.

2. The National Nosocomial Infections Surveillance (NNIS) System. Nosocomial infection rates for interhospital comparison: limitations and possible solution. Infect Control Hosp Epidemiol. 1991;12:609-21.

3. Archibald LK, Gaynes RP. Hospital-acquired infections in the United States: the importance of interhospital comparisons. Infect Dis Clin North Am. 1997;11:245-55. 
4. Knaus WA, Draper EA, Wagner DP, Zimmerman JE. APACHE II: a severity of disease classification system. Crit Care Med. $1985 ; 13: 818-29$.

5. The 2000 Report of Health and Labour Sciences Research Grant (Research on Emergent or Revival Infections) (in Japanese). Research on development of drug-resistant infection surveillance system, 2000.

6. Garner JS, Javis WR, Emori TG, Horan TC, Hughes JM. CDC definitions for nosocomial infections 1988. Am J Infect Control. 1988;16:128-40.

7. Suka M, Yoshida K, Takezawa J. Impact of intensive care unitacquired infection on hospital mortality in Japan: a multicenter cohort study. Environ Health Prev Med. 2004;9:53-7.

8. Suka M, Yoshida K, Takezawa J, Arakawa Y. Assessment of burden of ICU-acquired infections (in Japanese). Kankyo Kansen. 2004;19:389-94.

9. Bone RC, Balk RA, Cerra FB, Dellinger RP, Fein AM, Knaus WA, et al. Definitions for sepsis and organ failure and guidelines for the use of innovative therapies in sepsis. Chest. 1992;101:1644-55.

10. Levy MM, Fink MP, Marshall JC, Abraham E, Angus D, Cook D, et al. $2001 \mathrm{SCCM} / \mathrm{ESICM} / \mathrm{ACCP} / \mathrm{ATS} / \mathrm{SIS}$ international sepsis definitions conference. Crit Care Med. 2003;31:1250-6.

11. Suka M, Yoshida K, Takezawa J. Incidence and outcomes of sepsis in Japanese intensive care units: the Japanese Nosocomial Infection Surveillance System. Environ Health Prev Med. 2006;11:298-303.
12. Suka M, Yoshida K, Takezawa J. Association between APACHE II score and nosocomial infections in intensive care unit patients: a multicenter cohort study. Environ Health Prev Med. 2004;9:262-5.

13. Suka M, Yoshida K, Takezawa J. Association between APACHE II score and infection rates in ICU patients (in Japanese). Kankyo Kansen. 2005;20:200-4.

14. Fridkin SK, Welbel SF, Weinstein RA. Magnitude and prevention of nosocomial infections in the intensive care unit. Infect Dis Clin North Am. 1997;11:479-96.

15. Suka M, Yoshida K, Uno H, Takezawa J. Incidence and outcomes of ventilator-associated pneumonia in Japanese intensive care units: the Japanese Nosocomial Infection Surveillance System. Inf Cont Hosp Epidemiol. 2007;28:307-13.

16. Suka M, Yoshida K, Takezawa J. Organizational characteristics of intensive care units related to nosocomial infections: a multicenter study (in Japanese). Kankyo Kansen. 2005;20:24-30.

17. Suka M, Yoshida K, Takezawa J. Comparisons of denominators of nosocomial pneumonia rates in intensive care units: a multicenter study (in Japanese). Kankyo Kansen. 2005;20:133-8.

18. Suka M, Yoshida K, Takezawa J. A practical tool to assess the incidence of nosocomial infection in Japanese intensive care units: the Japanese Nosocomial Infection Surveillance System. J Hosp Inf. 2006;63:179-84.

19. Gustafson TL. Practical risk-adjusted quality control charts for infection control. Am J Infect Control. 2000;28:406-14. 